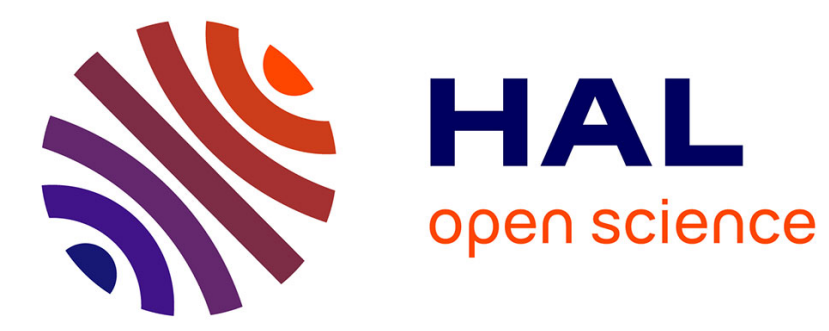

\title{
Improved 2 mrad crossing angle layout for the International Linear Collider
}

R. Appleby, D. Toprek, D. Angal-Kalinin, P. Bambade, S. Cavalier, O.

Dadoun

\section{- To cite this version:}

R. Appleby, D. Toprek, D. Angal-Kalinin, P. Bambade, S. Cavalier, et al.. Improved 2mrad crossing angle layout for the International Linear Collider. 22nd Particle Accelerator Conference 07 (PAC'07), Jun 2007, Albuquerque, United States. pp.THPMN077. in2p3-00168654

\section{HAL Id: in2p3-00168654 https://hal.in2p3.fr/in2p3-00168654}

Submitted on 29 Aug 2007

HAL is a multi-disciplinary open access archive for the deposit and dissemination of scientific research documents, whether they are published or not. The documents may come from teaching and research institutions in France or abroad, or from public or private research centers.
L'archive ouverte pluridisciplinaire HAL, est destinée au dépôt et à la diffusion de documents scientifiques de niveau recherche, publiés ou non, émanant des établissements d'enseignement et de recherche français ou étrangers, des laboratoires publics ou privés. 


\title{
IMPROVED 2 MRAD CROSSING ANGLE LAYOUT FOR THE INTERNATIONAL LINEAR COLLIDER
}

\author{
R. Appleby*, D. Toprek (The Cockcroft Institute and the University of Manchester, UK), \\ D. Angal-Kalinin (The Cockcroft Institute and ASTeC, UK), \\ P. Bambade, S. Cavalier, O. Dadoun (LAL, France)
}

\begin{abstract}
This paper describes the current status and plans of the on-going work to develop an alternative interaction region for the ILC with a 2 mrad crossing-angle. In the past year, a new design has been produced, following simpler concepts and assumptions in order to be as economical as possible ${ }^{1}$.
\end{abstract}

\section{MOTIVATION}

The 300 ns long ILC inter-bunch spacing allows choosing either large, small, or even null, crossing-angles at the interaction point (IP). Pros and cons exist for each. The main strength of the large $14 \mathrm{mrad}$ crossing-angle adopted as present baseline [1] is to allow independent magnetic channels for in and outgoing beams, by means of compact superconducting (SC) focusing magnets now under development. This eases the transport of the disrupted post-IP beams while facilitating adding diagnostics to serve precision physics studies, in particular a spectrometer and polarimeter complementing similar devices planned upstream in the Beam Delivery System (BDS).

Such a large crossing-angle however adds challenging constraints to the setup and tuning of the beams prior to collisions. To avoid large luminosity loss from the reduced bunch overlap, $\mathrm{x}-\mathrm{z}$ correlations (crab-crossing) are introduced through special RF cavities. A system of dipoles called DID/anti-DID is also added to the solenoid to locally cancel the horizontal field component seen by the beams traversing with a finite angle with respect to its axis. The trajectory perturbation from the non-axial geometry and DID/anti-DID must then be corrected before and after the IP. For the detector, a large crossing-angle is also disfavored as it complicates the very forward geometry, in particular for the calorimetric coverage at small polar angles. The DID/anti-DID, which may be set based on experimental conditions and requirements, also implies more complex and frequent track-based calibration procedures to monitor the resulting field distortions, which may limit the achievable track momentum resolution, e.g. if a Time Projection Chamber is used as main tracker.

For these reasons, alternative interaction region (IR) designs with small crossing-angles are pursued. Ideally, head-on collisions should be best [2]. The main challenge are then the electrostatic separators needed to extract the

\footnotetext{
*r.b.appleby@dl.ac.uk

${ }^{1}$ Work supported by the EC under the FP6 "Research Infrasctructure Action - Structuring the European Research Area" EUROTeV DS Project Contract no.011899 RIDS
}

spent beams. As a backup and potential alternative, the $2 \mathrm{mrad}$ scheme proposed at Snowmass [3] is reconsidered with a much shorter and economical design. The explicit aim is to use as few and feasible magnets as possible while accommodating all beam parameter sets specified for 0.5 and $1 \mathrm{TeV}$. The dedicated post-IP spectrometer and polarimeter chicanes planned in the $14 \mathrm{mrad}$ baseline design are excluded and one assumes instead that new methods, recently discussed to perform these measurements in different ways, will be successfully developed. Crab-crossing is also not relied on during initial ILC running (the corresponding luminosity loss is only $\sim 15 \%$ ) while space provisions are included to add it later as an upgrade. Finally, deflections from the off-axis geometry in the detector solenoid are small enough not to require a DID/anti-DID and induced pre and post-IP trajectory bumps are an orderof-magnitude smaller than for $14 \mathrm{mrad}$.

In the following, progress is reported on the new optical layout of the extraction line, for 0.5 and $1 \mathrm{TeV}$ centre-ofmass energies and including optimised final doublets (FD), on integration into the final focus and on designing the first bending magnet with shared aperture for spent and beamstrahlung beams. Plans to complete the design are outlined.

Studies of photon backscattering induced by particle losses in the extraction lines and of their detector impact pursued recently in the context of the 2 mrad design are reported in [4].

\section{PRESENT STATUS OF REDESIGN}

The layout of the new 2 mrad design is shown in Figure 1. Beamstrahlung cone clearances of 0.75 and 0.85 mrad half-opening angles were specified in each transverse plane [5] for the latest ILC beam parameters [6]. Beam loss calculations were done with very large statistics macroparticle files [7], to limit statistical errors to less than $1 \mathrm{~W}$.

\section{Final doublet}

The FDs are based on superconducting and warm technologies for the QD0-SD0 and QF1-SF1 magnet systems, respectively. For $0.5 \mathrm{TeV}$, QD0 uses parameters similar to the LHC main lattice design, based on NbTi and with a pole-tip field of $6.3 \mathrm{~T}$. For $1 \mathrm{TeV}, \mathrm{Nb}_{3} \mathrm{Sn}$ is planned with a pole-tip field of $8.8 \mathrm{~T}$. The maximum pole tip field for SD0 was taken to be $4.4 \mathrm{~T}$. For the QF1 and SF1 warm magnets, pole tip fields up to 1.4 and $0.75 \mathrm{~T}$ are used. The outgoing beam passes off-axis through the same apertures as the incoming beam in QD0 and SD0, where it is kicked out- 


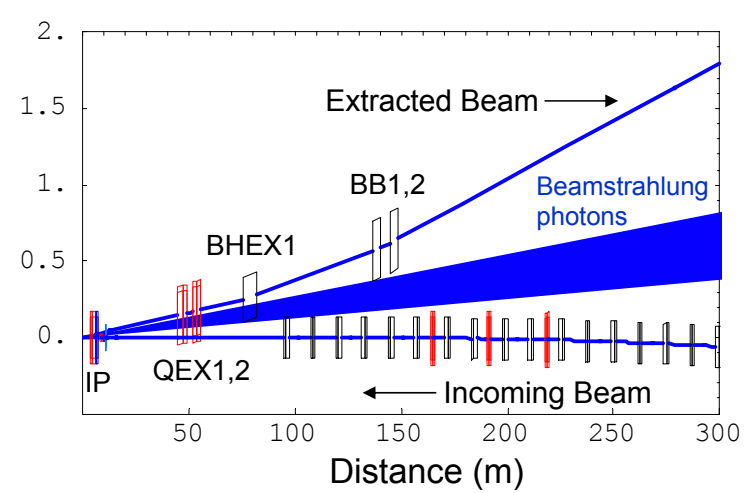

Figure 1: Layout of the new 2 mrad crossing-angle scheme. Bending magnets closest to the IP are protected against beamstrahlung tails by vertical collimators.

wards by about additional $2 \mathrm{mrad}$. This offsets it enough to move it into the pocket regions of QF1 and SF1, where fields and resulting inwards kicks are weaker. The magnitudes of the latter depend on apertures and offsets in QF1 and SF1. The parameter optimisation gave priority to ensure that less than $1 \mathrm{~W}$ total incident power was deposited in QD0 and SD0 (corresponding to a $0.5 \mathrm{~mW} / \mathrm{g}$ local power density tolerance in the SC material), for all ILC beam parameters and energies [8]. This resulted in compact designs with overall extraction angles after the FD reduced to about $3 \mathrm{mrad}$. Table 1 shows the quadrupole parameters for the $0.5 \mathrm{TeV}$ case. The full set can be found in [9].

Table 1: $0.5 \mathrm{TeV}$ final doublet quadrupole parameters.

\begin{tabular}{|l|c|c|}
\hline Parameter & QD0 & QF1 \\
\hline \hline Length $[\mathrm{m}]$ & 1.059 & 1.596 \\
\hline Strength & $-0.270 \mathrm{~m}^{-2}$ & $0.0786 \mathrm{~m}^{-2}$ \\
\hline radial aperture $[\mathrm{mm}]$ & 28 & 20 \\
\hline gradient $[\mathrm{T} / \mathrm{m}]$ & 225 & 65 \\
\hline
\end{tabular}

\section{Extraction line}

The extraction line starts after the shared final doublet region and has a total length of about $300 \mathrm{~m}$. $\beta$-functions and the dispersion are shown in Figure 2. After about $45 \mathrm{~m}$, two focusing quadrupoles centered on the outgoing beam and with apertures large enough to accommodate the beamstrahlung cone are used to extend the final doublet (QEX1) and to provide some weak vertical focusing (QEX2). Given the need to provide a quasi field-free region for the nearby incoming beam (offset only about $150 \mathrm{~mm}$ away from the centroid of the extracted beam), a Panofskystyle quadrupole design [10] is studied. Screening collimators rated up to $1 \mathrm{~kW}$ are included for protection.

The QEX1,2 magnet strengths are adjusted to minimise the disrupted beam size at the location of the first extraction bend BHEX1, a normal conducting C-shaped dipole with a $2 \mathrm{mrad}$ bend angle, $6 \mathrm{~m}$ length for $0.5 \mathrm{TeV}$ and orbit separation to the incoming beam of about $260 \mathrm{~mm}$. Since the overall angles of the extracted beamstrahlung and charged

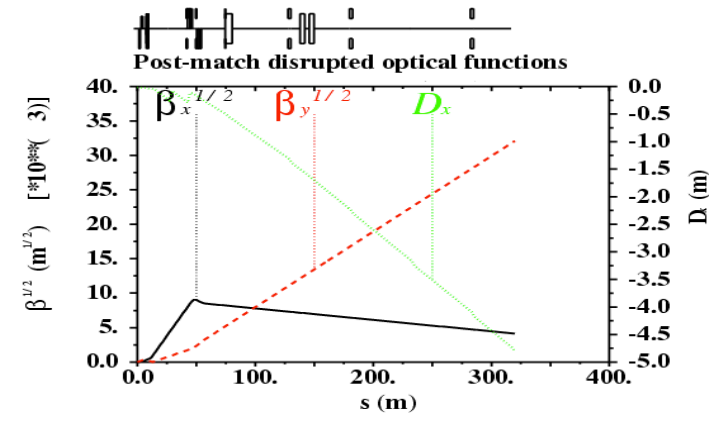

Figure 2: Optics for the $0.5 \mathrm{TeV}$ extraction line.

beams after the final doublet are about 2 and $3 \mathrm{mrad}$, respectively, its gap must be large enough to accommodate both, with final separation achieved only after. Collimators rated to $5 \mathrm{~kW}$ are included for protection against the low-energy beam and beamstrahlung tails. Two additional normal conducting dipoles, $\mathrm{BB} 1$ and $\mathrm{BB} 2$, are included after BHEX1 to provide the separation needed at the dump.

Primary collimators are designed to control the growth of the low-energy beam tail on the dump window. These collimators should absorb up to $210 \mathrm{~kW}$. A possible solution uses rotating $\mathrm{Al}$ balls in flowing water [11]. Collimator specifications for $0.5 \mathrm{teV}$ are listed in Table 2. The design is flexible such that beam sizes and the separation between in and outgoing beams at the dump location, about $320 \mathrm{~m}$ from the IP, can be adjusted easily to accommodate the different scenarios presently under consideration, with either completely shared, so-called co-joined or completely separate charged particle and photon dumps. The main changes involve the two bends BB1,2 and downstream collimator settings. The separations at $320 \mathrm{~m}$ needed for the three cases are $0.5,2$ and $3.5 \mathrm{~m}$, respectively. The second of these is realised in the optics presented, corresponding to $1.2 \mathrm{mrad}$ bend angles for $\mathrm{BB} 1,2$.

Table 2: Extraction line collimator losses at $500 \mathrm{GeV}$.

\begin{tabular}{|l|c|c|}
\hline Collimator & Length [m] & High Lumi loss [kW] \\
\hline \hline QEX1COLL & 1 & 0.2 \\
\hline QEX2COLL & 1 & 0 \\
\hline BHEX1COLL & 1 & 0.1 \\
\hline BB1COLL & 2.5 & 52.3 \\
\hline BB1COLL & 2.5 & 207.5 \\
\hline
\end{tabular}

\section{Final focus integration}

The extraction line final doublet optimisation was done using a simplified final focus system with two bends suitably placed upstream [8]. Integration into the ILC final focus is achieved by incorporating it into the baseline lattice [12]. The extra length can be absorbed at the location of the crab cavity in the RDR deck (in the 2 mrad case, provisional space for a future crab cavity upgrade will be 
far upstream). The local chromaticity correction is then re-optimised using BETA and LUMOPT [13]. The linear optics is first matched using dedicated quadrupoles and soft bending magnets. The sextupoles are then optimised to improve the optical bandwidth of the system. The result is shown in Figure 3. This is work in progress and improvements are expected using octupoles and decapoles, similar to those obtained in [14]. The strengths obtained for QD0, QF1, SD0 and SF1 are only marginally different from those in the simplified version and thus will not affect the losses and performance of the extraction line optics significantly.

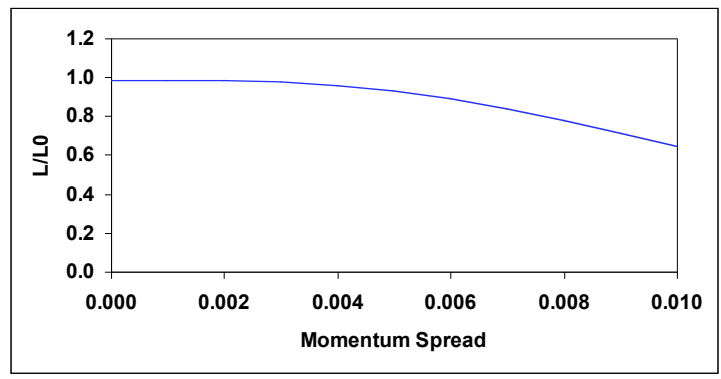

Figure 3: Optical bandwidth of the final focus for a $2 \mathrm{mrad}$ crossing-angle, using only sextupoles.

\section{BHEX1 design}

A $6 \mathrm{~cm}$ half-gap is needed in BHEX1 to accommodate the limited separation between the charged and beamstrahlung beams. The layout geometry and magnetic field computed with Poisson [15] are shown in Figures 4 and 5. Whilst this magnet does not present any particular technological problem, its large size and proximity to the incoming beam means that the field homogeneity inside and leakage field outside must be checked. The introduction of shims allows to limit the deviation of the vertical magnetic field component to less than $3.2 \%$ from its design value over the extent of the horizontal charged beam distribution. This was checked to have negligible effects on beam losses downstream. The leakage field at the location of the incoming beam is about $1 \%$ of the design field inside the magnet. This results in a sizeable $20 \mu \mathrm{rad}$ kick (corresponding to $\sim 7.5$ times the incoming beam angular divergence) which must be corrected nearby. Furthermore, the effect of the residual vertical decapole field component has been incorporated into the simplified model of the final focus, yielding negligible effects on the vertical beam size at the IP. Further more complete checks are planned with the complete final focus.

\section{CONCLUSIONS}

In this paper, an improved $2 \mathrm{mrad}$ crossing angle scheme has been presented, including carefully optimized FDs, integration into the final focus and design of the first bending magnet with shared aperture for the spent and beamstrahlung beams. The new layout has considerable advan-

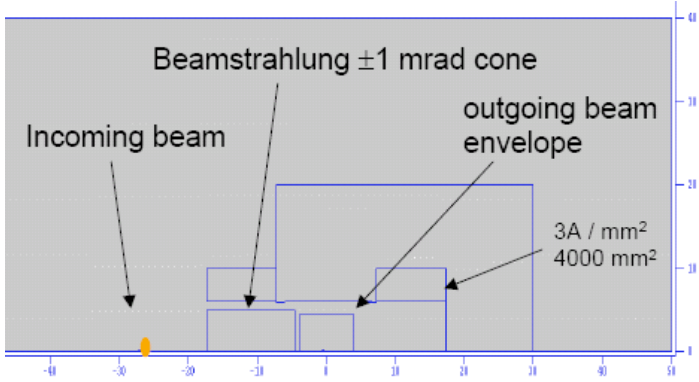

Figure 4: Geometry of the BHEX1 extraction line magnet.

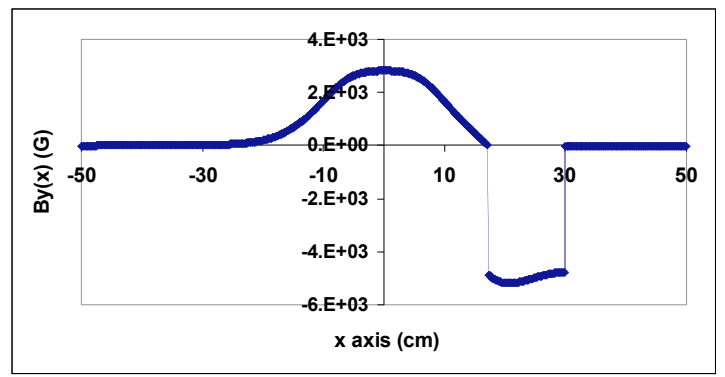

Figure 5: On-axis BHEX1 $\mathrm{B}_{\mathrm{y}}(x)$ field around the spent beam reference trajectory. Units are in $\mathrm{cm}$ and Gauss.

tages over previous schemes. The plan for the EDR phase of the project are to develop the magnet and beam pipe designs, continue evaluating IP backgrounds and study and integrate relevant post-IP instrumentation, in order to provide a competitive alternative to the baseline design.

\section{REFERENCES}

[1] A. Seryi et al, Design of the Beam Delivery System for the International Linear Collider, in these Proceedings.

[2] O. Napoly et al, Technical Challenges for Head-On Collisions and Extraction at the ILC, in these Proceedings.

[3] R. Appleby et al, EuroTeV report 2006-001-01.

[4] O. Dadoun et al, Backscattering of Secondary Particles into the ILC Detectors from Beam Losses along the Extraction Lines, in these Proceedings.

[5] R. Appleby el al, EuroTeV report 2006-001-14, Under preparation.

[6] see http://media.linearcollider.org/report-apr03-part1.pdf

[7] http://flc-mdi.lal.in2p3.fr/spip.php?rubrique17

[8] R. Appleby and P. Bambade, JINST 1 P10005 (2006), EuroTeV report-2006-022, physics/0606022.

[9] R. Appleby el al, EuroTeV memo 2007-001-01.

[10] L. Hand and W. Panofsky, Rev.Sci.Instrum.30:927,1959.

[11] Lew Keller, Private Communication.

[12] ILC-report-2007-01

[13] J. Payet $a l$, Automatic ILC Luminosity Optimisation, in these Proceedings.

[14] F. Jackson et al, Collimation optimisation in the beam delivery system of the ILC, in these Proceedings.

[15] POISSON, LA-UR-96-1834. 